\title{
Development of a methodology for the application of the Warren-Averbach method
}

\author{
Ichikawa, R. U. ${ }^{1}$; Martinez, L.G. ${ }^{1}$; Imakuma, K. ${ }^{1}$; Turrillas, X. ${ }^{2}$ \\ 1 Nuclear and Energy Research Institute, Materials Science and Technology Center, São Paulo, SP, Brazil \\ 2 Institute of Materials Science of Barcelona, Department of Crystallography, Bellaterra, Spain
}

\begin{abstract}
The objective of this work was the development of a methodology to speed up the application of the Warren-Averbach method of X-ray line profile analysis to determine the mean crystallite size and microstrain in polycrystalline materials. A computer program was developed in Python programming language to implement a Discrete Fourier Transform (DFT) algorithm instead of other such as the Fast Fourier Transform, and then used to apply the Stokes deconvolution method to correct the instrumental contribution in the X-ray profiles.
\end{abstract}

Keywords (Palavras chaves): Warren-Averbach method, computer program, X-ray line profile analysis.

\section{Introduction}

Crystallographers were the early scientists to employ the use of computers in their studies when they became research tools in the 1950s [1]. Their uses were limited since the low memory, low speed and few computer languages restricted their application. According to the advance of the languages and hardware, the development of computer programs began rapidly to spread. In this era of development it was common that computer programs were freely distributed as a form to enrich the discussion about them and also to improve it. Nowadays, however, freeware programs are hardly well documented and updated. A few exceptions include DBWS [2], GSAS [3] and FullProf [4], the principal Rietveld refinement softwares used worldwide.

But when microstructural analysis needs to be performed an even smaller number of programs can be found. An exception may be cited as an example, the PM2K [5], a well-known and powerful program for microstructural characterization of nanocrystalline materials. In order improve the softwares dedicated to microstructural studies, in this work a program that speed up the application of the Warren-Averbach [6] method is presented.

The microstructure of materials can be characterized by many factors including size and form of its structure, strains, dislocations, stacking faults, grain boundaries, etc. And how to relate these factors to properties of microstructure has been one of the most important subjects of study in materials science [7]. This work focused on the application of the Warren-Averbach method that allows us to determine the mean crystallite size (MCS) of the microstructure and its microstrain. The evaluation of MCS it is of major importance since it can be used to certificate a material as nanocrystalline. Also as discussed by Krill et al. [7] an important characteristic in majority of physics concepts it is that characteristics which depends on length, such as the electron mean free path $l$ can be related with MSC which can be related to electrical conductivity of a material. When MCS it is greater than $l$ the electrical conductivity can be studied modeling the microstructure of the material as a network of interconnected resistors.

In this work the Warren-Averbach method [6] was studied in order to determine the mean crystallite size and the microstrain in materials. This method is one of the most powerful methods for the separation of these two contributions in X-ray diffraction profiles, since complete information can be obtained without make any assumptions on the shape of the profiles. This is performed expressing the diffraction peak as a Fourier series and from its coefficients (and also with at least two parallel reflections) the two contributions can be determined. Also a discussion about the separation of mean crystallite size and microstrain from the Fourier coefficients is made. It was seen that the separation of both contributions could be performed more easily if a fitting procedure is carried out on the graph of $A(L)$ vs L. 


\section{Methodology}

\subsection{Discrete Fourier Transform}

In this work, an algorithm for the Discrete Fourier Transform application was developed instead of using the widely known Fast Fourier Transform algorithm. This development has provided more freedom in the initial conditions, e.g. is not necessary that the number of points $(\mathrm{N})$ always be a power of 2 , as required by the FFT algorithm.

For the developed algorithm, the straightforward definition for the DFT was considered [8].

$$
F(u)=\frac{1}{N} \sum_{x=0}^{N-1} f(x) e^{-i 2 \pi u x / N}
$$

Where $u=0,1,2,3, \ldots N-1$.

To simplify the data output, the input data was considered as a non-causal signal, where the definition of causal and non-causal signal is given by [9]:

- A signal $f(x)$ is said to be causal, if it is defined for $x \geq 0$

- A signal $f(x)$ is said to be non-causal, if it is defined for both $x \leq 0$ and $x>0$.
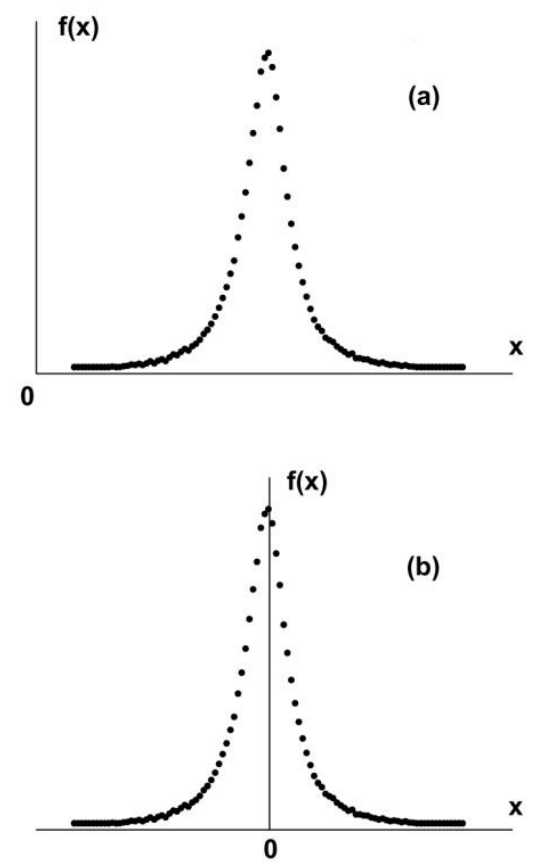

Figure 1 - Example of (a) a causal signal and (b) a non-causal signal.

In FIG. 1 it can be seen an example of a diffraction peak considered as a causal and non-causal signal.

\subsection{Warren-Averbach method}

The X-ray diffraction profile can be represented as a Fourier series in the reciprocal space [6]:

$$
\begin{gathered}
P(2 \theta)=\frac{K N F^{2}}{\operatorname{sen}^{2} \theta} \sum_{n=-\infty}^{\infty}\left[A_{n} \cos (2 \pi n h)+\right. \\
\left.B_{n} \operatorname{sen}(2 \pi n h)\right]
\end{gathered}
$$

Where $P(2 \theta)$ is the measured diffraction profile for $2 \theta$, $F$ is the structure factor and $K$ is the angular factor. $N$ represents the number of unit cells in the analyzed material and $n$ is the harmonic number.

The real Fourier coefficient is the product of two terms, one dependent on the length of columns of unit cells $[6,10]$ and therefore related to the crystallite size and another related to deformation in the crystal lattice and which depends on $1 / d$, where $d$ is the interplanar distance of the analyzed material, dependent therefore on the order of the reflection of the profile considered. The real coefficient can be written as:

$$
A\left(L, \frac{1}{d}\right)=A^{S}(L) \cdot A^{D}\left(L, \frac{1}{d}\right)
$$

It is therefore possible to separate the two coefficients, since the coefficient related to deformation $\left(\varepsilon_{L}\right)$ depends on the order of reflection and can be written as $\left\langle\cos 2 \pi \varepsilon_{L} L / d\right\rangle$ [4]. Thus if assuming that $L$ tends to zero, the following approximation can be considered:

$$
\left\langle\cos 2 \pi \varepsilon_{L} L / d\right\rangle \rightarrow 1-2 \pi^{2}\left\langle\varepsilon_{L}{ }^{2}\right\rangle L^{2} / d^{2}
$$

Replacing into Eq. 3 and applying the logarithm on both sides of the equation we get:

$$
\ln A\left(L, \frac{1}{d}\right)=\ln A^{S}(L)-2 \pi^{2}\left\langle\varepsilon_{L}^{2}\right\rangle L^{2} / d^{2}
$$

Where $L$ is termed as Fourier Length and is given by:

$$
L=\frac{n \lambda}{2\left(\operatorname{sen} \theta_{2}-\operatorname{sen} \theta_{1}\right)}
$$

Where $n$ is the harmonic number of the Fourier Transform and $\theta_{2}$ and $\theta_{1}$ are the angles that correspond to the initial and final angle of the diffraction peak considered and $\left\langle\varepsilon_{L}{ }^{2}\right\rangle$ is the mean square strain related to $L$ and can be used to calculate the root mean square strain $\left(\sqrt{\left\langle\varepsilon_{L}{ }^{2}\right\rangle}, \mathrm{RMSS}\right)$ which is more used. $\mathrm{A}$ detailed description of different types of microstrain representations can be found in references [11,12].

Therefore, using multiple orders of reflection (at least two) it is possible to separate the two coefficients on a graph of $\ln A\left(L, \frac{1}{d}\right)$ versus $\left(\frac{1}{d}\right)^{2}$. The coefficient related to the size $A^{S}(L)$ is obtained by the linear coefficient of linear fit on the graph and $\left\langle\varepsilon_{L}{ }^{2}\right\rangle$ can be obtained from the slope. 


\section{Blucher Proceedings \\ IV Workshop of Applied Crystallography to \\ Materials Science and Engineering}

To determine the crystallite size we can plot $A^{S}(L)$ versus $L$. The intersection of a straight line to the initial slope of the curve in the graph on the $L$ axis gives the crystallite size weighted by the area $\langle L\rangle_{A}$ (areaweighted mean crystallite size) [6].

\section{Results}

\subsection{Peak treatment guideline}

For the application of the Warren-Averbach method in this work a guideline for the peak treatment was followed. In a previous work by Ichikawa [11] and Martinez [13] this guideline was followed and shown to be appropriate to speed up and optimize the method.

The first treatment is not mandatory, consists in a peak smoothing if the data statistics is not adequate, for instance in cases where the peak does not present ideal conditions (low counting time or for low intensity reflections, which may cause noisy data), since its use can affect the peak shape. The smoothing is performed using the well-known Saviztky-Golay algorithm [14] or a method of adjacent points, in the latter procedure a mean value is calculated considering 3,5 or 7 points successively. For the background correction a simple linear regression is performed on the "tails" on both sides of the peak, and then subtracted. The intensities on these "tails" may assume negative intensity values, so when this occurs its intensity is brought to zero. At this point the Lorentz-Polarization correction can be performed to take into account the scattering of nonpolarized wave [15]. The final steps are the centralization and normalization of the peak as required by the DFT algorithm. Then the DFT can finally be executed. The Stokes method [16] is applied considering the results of DFT application in a standard reference material and in the material analyzed.

\subsection{Polynomial fit in the Fourier coefficients}

For the calculation of mean crystallite size and microstrain using the Warren-Averbach method, a conversion of harmonic number to Fourier length needs to be carried out. However when this conversion is performed the discretization of the harmonic numbers is lost for parallel reflections considered in the analysis, since the Fourier length is evaluated as showed by Eq. 4 where the parameter $\left(\operatorname{sen} \theta_{2}-\operatorname{sen} \theta_{1}\right)$ is diferent (and is not a multiple) according to the parallel reflections.

To overcome this problem a polynomial fitting procedure in the Fourier coefficients were performed. To evaluate the fitting procedure a parameter termed as coefficient of determination $\left(\mathrm{R}^{2}\right)$ was considered. This coefficient is used as a measure of quality of the fitting and a close value to 1 indicates a good fit.
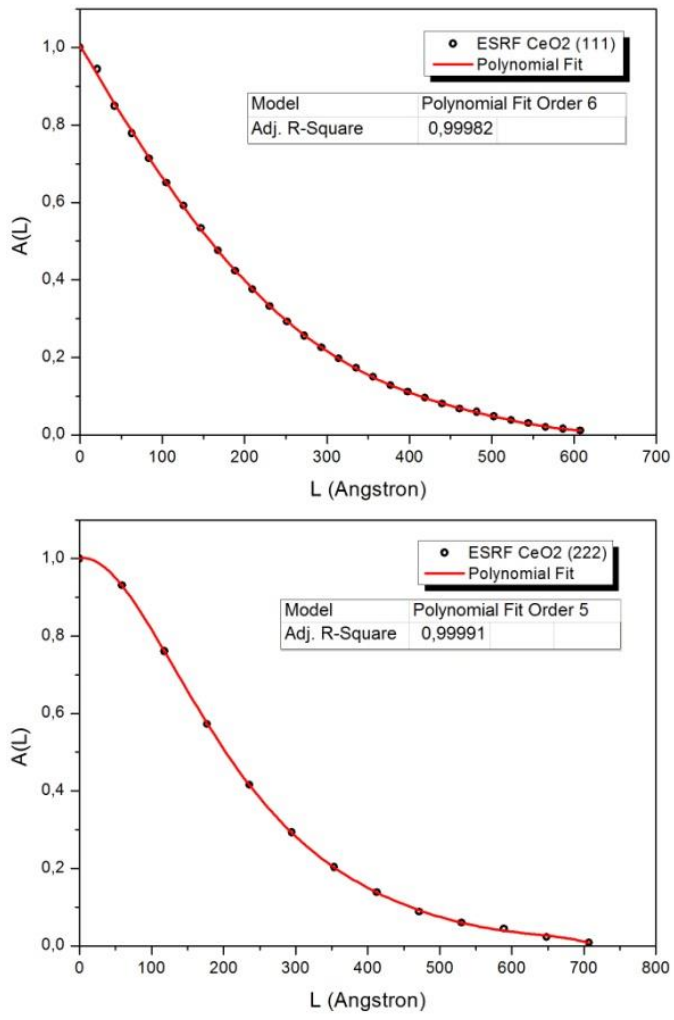

Figure 2 - Fitting procedure in the Fourier coefficients for the ESRF dataset of the of $\mathrm{CeO} 2$, Round-Robin collective work [12].

To confirm the proposed fitting procedure, a powder diffraction dataset of $\mathrm{CeO}_{2}$, published in a Round-Robin collective work [12] was analyzed according to the guideline discussed in Section 3.2. In Tab. 1 the results can be seen.

Table 1 - Values for $\mathrm{R}^{2}$ for 12 polynomial fits performed for the dataset of the of $\mathrm{CeO}_{2}$, Round-Robin collective work [12].

\begin{tabular}{ccccc}
\hline & \multicolumn{2}{c}{$(\mathbf{1 1 1 )}$} & \multicolumn{2}{c}{$(\mathbf{2 2 2})$} \\
\hline Dataset & $\begin{array}{c}\text { Pol. } \\
\text { Order }\end{array}$ & $\mathbf{R}^{\mathbf{2}}$ & $\begin{array}{c}\text { Pol. } \\
\text { Order }\end{array}$ & $\mathbf{R}^{\mathbf{2}}$ \\
\hline Birmingham & 5 & 0.99947 & 5 & 0.99894 \\
Le Mans & 6 & 0.99983 & 5 & 0.99935 \\
ESRF & 6 & 0.99982 & 6 & 0.99991 \\
NSLS & 6 & 0.99965 & 6 & 0.99974 \\
ILL & 6 & 0.99883 & 5 & 0.99998 \\
NIST & 6 & 0.99812 & 6 & 0.99992 \\
\hline
\end{tabular}

Analyzing the two parallel reflections (111) and (222) of the dataset [12] it was verified that polynomials of 5th and 6th orders were the ones with best results for the coefficient of determination $\left(R^{2}\right)$.

\subsection{Validation of the results}

With the polynomial equation obtained, equal values for the Fourier length can be used to apply the separation method according to Eq. 2 and the plot of Fourier coefficients related to the size, $A^{S}(L)$ versus $L$ can be 
used to calculate the area-weighted mean crystallite size $\left(\langle L\rangle_{A}\right)$.

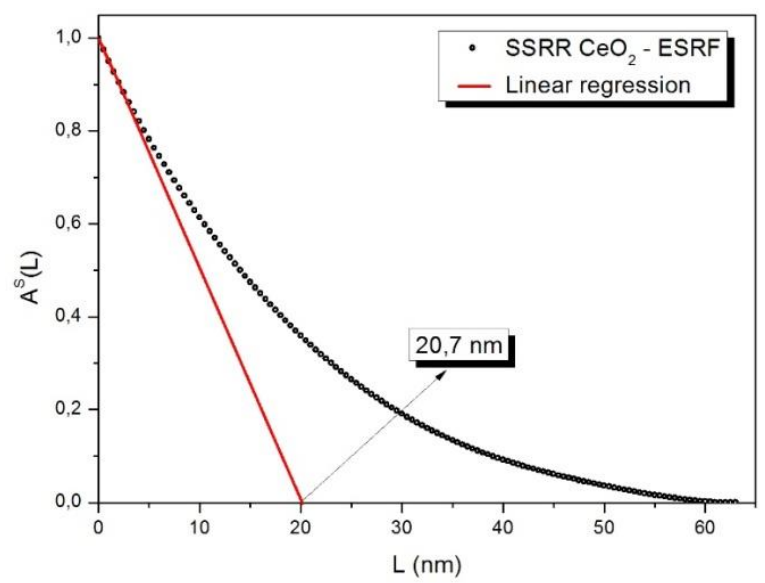

Figure 4 - Plot of $A^{S}(L)$ versus $L$ using the ESRF dataset of the of $\mathrm{CeO} 2$, Round-Robin collective work [12] for the calculation of $\langle L\rangle_{A}$.

The results for the $\langle L\rangle_{A}$ and RMSS using the RoundRobin data are presented in Tab. 2.

Table 2 - Comparison of the results for the $\langle L\rangle \_A$ and RMSS.

\begin{tabular}{ccccc}
\hline & \multicolumn{2}{c}{$(\mathbf{1 1 1})$} & \multicolumn{2}{c}{$(\mathbf{2 2 2})$} \\
\hline Dataset & $\begin{array}{c}\text { LA } \\
\text { (nm) }\end{array}$ & RMSS & $\begin{array}{c}\text { LA } \\
\text { (nm) }\end{array}$ & RMSS \\
\hline Birmingham & 20,5 & $2 \mathrm{E}-06$ & 17,7 & 4,4 \\
Le Mans & 18,8 & $0^{*}$ & 19,8 & 6,6 \\
ESRF & 20,7 & $0^{*}$ & 19,5 & 0 * \\
NSLS & 19,9 & $0^{*}$ & 19,6 & 4,1 \\
ILL & 21,1 & $0^{*}$ & 18,8 & 4,5 \\
NIST & 20,9 & $0^{*}$ & 19,4 & 7,1 \\
\hline
\end{tabular}

For a better comparison, the average for the values presented in Tab. 2 was calculated. For this work it was obtained an average value of $20,33 \pm 0,85 \mathrm{~nm}$ and $19,13 \pm 0,78 \mathrm{~nm}$ considering the results applying the Warren-Averbach method. For the RMSS it was reported in the Round-Robin work [17] that these values are extremely low and can even be neglected.

\section{Conclusions}

It can be seen that the mean values obtained comparing this work with the $\mathrm{CeO}_{2}$ Round-Robin work did not significantly differ from each other. The difference can be explained due to the methodology proposed in this work, where simpler treatments, e.g. the background correction and the Stokes deconvolution were considered. As for the RMSS, in the SSRR work it was considered that the magnitude of the values are relatively small and can be neglected. It can be concluded that the values are comparable considering the standard deviation. Since the objective of this work was to propose a methodology to simplify and speed up the application of the Warren-Averbach method the results shown satisfactory results for its purpose.

\section{References}

[1] SMITH, F.: Industrial Applications of X-Ray Diffraction. CRC Press. 1999.

[2] YOUNG, R. A., et al.: User's Guide to Program DBWS-9807a for Rietveld Analysis of X-ray and Neutron Powder Diffraction Patterns. School of Physics, Georgia Institute of Technology, Atlanta GA, USA. 1999.

[3] TOBY, B. H. J. Appl. Cryst., v. 34, p. 210-213, 2001.

[4] RODRIGUEZ-CARVAJAL, J. FULLPROF, Reference Guide, Laboratoire Leon Brillouin (CEACNRS), 1994.

[5] LEONI, M. Z. Kristallogr. Suppl., v. 23, p. 249-254, 2006.

[6] WARREN, B. E.; AVERBACH, B. L. J. Appl. Phys., v. 21 , p. $595-599,1950$.

[7] KRILL, C. E.; BIRRINGER, R. Philos. Mag. A, v. 77, p. $621-640,1998$.

[8] ARFKEN, G. B.; Weber, H. J.: Mathematical Methods for Physicists. Academic Press. 2005

[9] KANI, A. N.: Signals and Systems. Tata McGraw Hill. New Delhi. 2010.

[10] BERTAUT, F. C. R. Acad. Sci. Paris., v. 228, p. 187-189, 1949.

[11] ICHIKAWA, R. U., M.Sc. Dissertation, University of São Paulo, São Paulo, Brazil, 2013.

[12] BALZAR, D. and LEDBETTER, H.: J. Appl. Cryst., v. 26, p. 97-103, 1993.

[13] MARTINEZ, L. G., M.Sc. Dissertation, University of São Paulo, São Paulo, Brazil, 1989.

[14] SAVITZKY, A.; GOLAY, M. J. E. Anal. Chem., v. 36, p. 1627-1639, 1964.

[15] KLUG, H. P.; ALEXANDER, L. E.: X-ray Diffraction Procedures: For Polycrystalline and Amorphous Materials, John Wiley \& Sons, New York, NY, USA, 2nd edition, 1974.

[16] STOKES, A. R., Proc. Phys. Soc., v. 61, p. 382391, 1948.

[17] BALZAR, D. et al., J. Appl. Cryst., v. 37 p. 911- , 2004. 